\title{
Ultra Wideband Assisted Localization of Semi-Autonomous Floor Scrubber
}

\author{
Višeslav Čelan, Ivo Stančić, Member, IEEE, and Josip Musić*, Member, IEEE
}

\begin{abstract}
The paper describes the design and features of the novel semi-autonomous floor scrubber add-on module, used for cleaning large indoor spaces. Module is designed in such a manner that it can be easily attached and detached from scrubber machine and that additional sensors can be introduced if needed. The paper focuses on the localization capabilities of the machine in several sensor setups with emphasis on the use of ultra wideband (UWB) real-time localization system (RTLS). It also proposes fusion of sensor data from several sources including novel use of wheel encoder's data in UWB setup. Analysis is performed in terms of localization accuracy and reliability as well as associated advantages and disadvantages. Obtained results demonstrated that inclusion of UWB subsystem, despite its price and accuracy ( $\pm 20 \mathrm{~cm}$ in ideal, line of sight, conditions), based on behavior switching yields more reliable and accurate results in open spaces (up to 25 times in position and 2 times in orientation) and that its accuracy can be further improved with inclusion of wheel encoder data.
\end{abstract}

Index Terms-UWB, robot localization, navigation, EKF, service robotics, sensor fusion.

\section{INTRODUCTION}

Modern living spaces like shopping malls, offices, schools, universities, hospitals, airports, etc., regardless of their size and configuration, have number of rooms within them that require constant care and cleaning. This is usually achieved with specialized machinery and trained personnel either during work hours or outside it depending on structure and purpose of the space. This can in turn interfere with normal functioning of the space and takes up resources that could otherwise be better utilized. Labor shortage is also an important factor that should be taken into account [1]. Thus, if this task could be automatized, optimized and performed during off-work hours (or at least during off-peak hours) improved user experience, increased safety and savings could potentiality be achieved. Additionally, if this transition from non-automatized to automatized approach could be managed in economical manner by using already available floor scrubbers with implementation of modular approach it could be attractive to wide(r) audience.

In recent years, because of reduction in size and cost of sensors and off-the-shelf embedded computers, as well as

Manuscript received January 13, 2017; revised May 12, 2017. Date of publication June 1, 2017.

V. Čelan was with Faculty of Electrical Engineering, Mechanical Engineering and Naval Architecture, University of Split, Split, Croatia and is now with Statim d.o.o., Split, Croatia (e-mail: viseslav.celan@gmail.com).

I. Stančić and J. Musić are with Faculty of Electrical Engineering, Mechanical Engineering and Naval Architecture, University of Split, Split, Croatia (e-mails: \{Ivo.Stancic, Josip.Music\}@fesb.hr).

Digital Object Identifier (DOI): 10.24138/jcomss.v13i2.379

* Corresponding author. increase in their computational power and reliability, service robotics has grown significantly and is becoming ubiquitous. According to ISO 8373- 2012 standard [2] a service robot is a robot that performs some task useful to humans (or other equipment) excluding industrial application(s). Investments in robotics as a field grew about 240 million USD in 2015 and they account for about a third of all investments in HAX startups [3]. Other studies [4], [5] noted that service robots will reach a global market volume equivalent to industrial robots (which is estimated to be over 15 billion Euros) between 2020 and 2025. This has been additionally fueled by software developments like Willow Garage's Robotic Operating System (ROS) [6], which is becoming increasingly used by researchers and private companies alike.

The aim of the research presented in the paper is to produce add-on module that would transform standard floor scrubber to autonomous one and that can, in case of need, be easily transferred to other machine, or upgraded. The robot would still need human intervention for filling up water tank and cleaning agent and subsequent disposal of dirty water. In the process special focus is given to scrubber localization in known spaces through proposal of innovative applications of UWB RTLS system (which currently is not widely used in robotics) and other sensors like lidar or wheel encoders. The work was originally published within SpliTech 2016 [7] but is now significantly expanded with inclusion of cases with fusion of data from wheel encoders and referent measurements with camera system (for comparison purposes; it required new measurement setup, new measurements as well as new data analysis). Also number of additional explanations and improvements have been included throughout the text as well as new references, especially in Related work section.

\section{RELATED WORK}

Specialized autonomous industrial sized floor scrubbing robots already exist as complete products [8] and in some cases in advanced stages of the development stage [9]. Unfortunately their price is usually much higher than regular industry size scrubbers. On smaller scale, Roomba is probably the best known example of the autonomous service robot [10] although other similar products exist. It was firstly introduced in 2002 and up to February 2014 over 10 million units were sold. Recently, model 980 which uses a camera for vision Simultaneous Localization and Mapping (VSLAM) [11], was introduced. Similar products also use localization for increased 
cleaning efficiency but relay on different hardware like Neato XV-11 from Neato Robotics [12].

More recent developments in the field of service robotics include Fetch and Freight robots from Fetch Robotics [13]. It is worth noting that both Fetch and Freight are based on ROS. The development of the robot pair (i.e. manipulator and moving base) is motivated by the fact that there are estimated 600,000 unfilled jobs in the logistics industry, and that ecommerce is expanding industry with sales increasing by about $15 \%$ to around 300 billion USD in 2014. Similar product exist from other manufacturers like OTTO robot from Clearpath Robotics [14]. Another service robot that uses ROS for its operation is Relay from Savioke [3]. The robot is aimed at hospitality services like hotels and acts as a room service delivery tool. It has been already implemented in several hotels like Crown Plaza Silicon Valley, San Jose, USA and Marriot, Los Angeles, USA. Other service robot products include Dispatch (open space delivery robot) from Distpatch robotics [15], FellowTwo (retail store assistant) from Fellow Robots [16], Adept robots (mutifunctional service robots and mobile base) from Adept Technology Inc. [17] and Care-O-bot 4 (multipurpose service robot and robot bases) from Fraunhofer Institute Manufacturing Engineering and Automation [18]. It is interesting to note (especially for service robotics) that in [19] it has been observed that behavioral and social norms are expected from the robots in typical retail type environment, but (at the same time) not from the human user.

Localization plays an important role in (semi)autonomous service robots. In [20] a stigmergic approach was used in conjunction with RFID-based navigation maps in goal-directed navigation of full-scale robots like Scitios G5 and Turtlebot. Robots navigated in $80 \mathrm{~m}^{2}$ apartments which were part of a larger residential area for senior citizens [21]. In [22] possibility of application of mobile robots to move materials around a hospital was examined. Semi-autonomous mobile robots were suggested as possible solution relaying on visual tags (ID based augmented reality markers and 2D barcodes) due to their ease of installation, low cost and constructional simplicity. Range of localization methods were tested including barometric approach for multi-floor buildings and semantic annotation as well as GPU based visual approach. Besides hospitals, robots can be used to provide care for elderly in their homes [23] or as means for an extended telepresence of medical professional [24]. Mobile robot navigation was also in focus in [25] for applications such as transportation, cleaning, search and rescue and surveillance. The work presents several approaches to efficiently estimate state of the robot while performing SLAM as well as estimate of the model of the environment (including highly complex and dynamic environments). The proposed approach was successfully tested in two practical applications: 1.) parking a car in a complex multi-level urban environment with no (or poor) GPS signal, and 2.) navigation of a robotic pedestrian assistant in largescale, highly complex and dynamic environment. Service robot navigation systems for similar applications in indoor environments [26] and in highly structured outdoor environments (like vineyards and orchards) [27] are being also developed.

Ultra wideband real time localization systems [28] are becoming increasingly available and relatively affordable with localization accuracy ranging from several $\mathrm{cms}$ to several dozen $\mathrm{cms}$ depending on measurement conditions and particular system. They are kind of GPS system giving absolute location, where so called anchors (antennas) are analogous to satellites and UWB tags are similar to GPS receivers. They can be used both indoors and outdoors, and are based on time-offlight measurements. Another often important shortcoming is low sampling frequency of UWB RTLS (usually around 2-10 $\mathrm{Hz}$, depending on number of tags). Number of researcher have tried to increase reliability and accuracy of such systems with inclusion of other types of sensor like inertial sensors or with modeling of measurement noise [29]. In [30] authors used tightly coupled approach using UWB system, accelerometer and gyroscope in order to estimate accurate indoor position. In order to achieve this, sensor models were developed including UWB measurement model based on combination of heavytailed Cauchy distribution and Gaussian distribution. Sensor fusion was achieved using maximum a posteriori (MAP) estimation algorithm. Obtained results (in comparison to referent optical system - Vicon) demonstrated drift free pose estimate with root mean square error (RMSE) of $3 \mathrm{~cm}$ in position and less than $1^{\circ}$ in orientation. Same sensor setup (UWB and IMU) was used in [31] but with different data fusion algorithm. Here lose coupling was used via Kalman filtering approach. Loose coupling (as opposed to tight coupling used in [30]) refers to the fact that each system operates independently from the other and their outputs (measurements) are fused together afterwards. This approach yielded global transnational error of $0.14 \mathrm{~m}$ as opposed to the same error of $0.56 \mathrm{~m}$ when only IMU system was used. It is also worth noting that this approach resulted in increase of sampling frequency which was just $6 \mathrm{~Hz}$ for UWB system to $30 \mathrm{~Hz}$ (equal to the frequency of IMU system). In outdoor environment additional sensors like GPS can be added to the setup. This was done in [32] in tightly coupled fashion using robust Kalman filter. Experimental results confirmed significant improvement in such scenario as compared to other scenarios like GPS only based localization. In [33] combination of UWB based system (UWB radar) and lidar was explored in conjunction with camera system. The main idea in that particular research was to combine lidar and camera systems from improved obstacle avoidance while behavior-based sensor fusion was used to switch to UWB radar based obstacle avoidance in cases when environmental conditions (e.g. fog) changed. The approach was tested successfully using mobile robot in several scenarios.

In the paper we propose use of novel combination of UWB localization system and robot based sensors (i.e. lidar and wheel encoders) for improved accuracy through data fusion based on customized extended Kalman filter (EKF). This in conjunction with behavior-based switching contributes to system reliability in practical applications.

\section{MATERIALS AND MethodS}

The proposed add-on module consists of three main parts: mechanical construction, electronics (including drive train, 


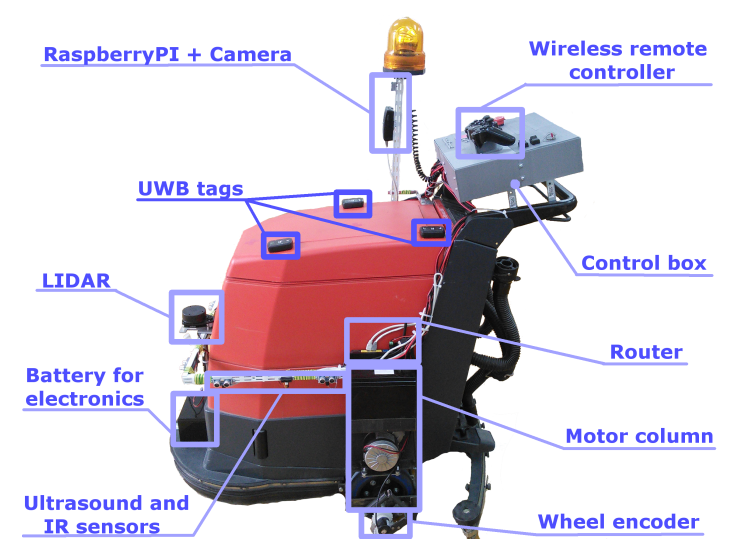

Figure 1. Proposed semi-autonomous system with highlighted individual components of the add-on module.

sensors and controllers), and software with control algorithms. For testing purposes it was mounted on Hakomatic E 530 floor scrubber which measures $123 \mathrm{~cm}$ in length and $53 \mathrm{~cm}$ in width. The machine weighs in (without the add-on module but with full water tank) around $200 \mathrm{~kg}$ and can travel with speeds up to $5 \mathrm{~km} / \mathrm{h}$. It has its own separate $24 \mathrm{~V}$ battery pack that powers scrubber brushes as well as vacuum extension that picks up excess water from the floor.

It is worth mentioning that the total cost of the system (which includes previously mentioned scrubber) is around 9.000 Euros (not including any profit margin), while similar commercially available systems like the ones from Taski Intellibot and CleanFix cost between 13.000 and 26.000 Euros. The comparison might not be completely fair since commercially available systems do not have UWB based localization, but do have other features that our system currently does not have.

\section{A. Mechanical construction}

Main part of the module is $4 \mathrm{~mm}$ thick steel construction in form of two columns, each placed on one side of a floor scrubber driving one wheel. Each column is a three level construction that holds the drive train (DC motor), drive wheel with wheel encoder, and power supply (12 V 25 Ah deep cycle battery) and is depicted in Figure 1. CAD (computeraided design) drawing in Figure 2 is also provided for easier understanding. It is attached to the floor scrubber via two bolts (per motor column), which are easy to undo in case of module removal. This is possible due to a metal beam added to the undercarriage of the floor scrubber. The beam is not visible from outside/above and it does not interfere with normal/manual operation of the scrubber (in the case when add-on module is removed). That was the only change made to the original machine. All other components were attached to the machine via already available bolt/screw holes.

Each drive motor is connected to the drive wheel (i.e. additional wheel and not the scrubber's original wheel) via chain drive and gears. In conjunction with the drive wheel, smaller wheel with its own gear system was added and was attached to the encoder. In this manner possible drive wheel slips would not be measured by the encoder and increased accuracy and

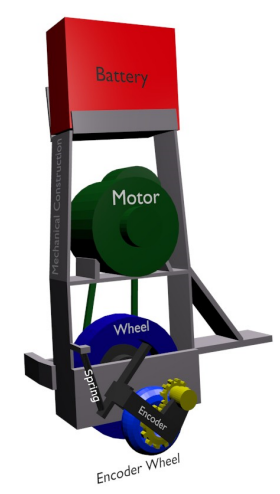

Figure 2. Mechanical construction of a single motor column. Two columns are needed per floor scrubber.

reliability levels are achieved. Additionally, better friction of the encoder wheel was ensured by attaching a strong metal spring so the whole encoder structure is firmly pressed to the floor. This can be seen more clearly in Figure 2.

\section{B. Electronics}

Electronic components in current prototype are placed in a single $3 \mathrm{D}$ printed case, which is mounted on the scrubber handles using existing wholes and screws. Except drive train, only distance sensors (e.g. lidar and ultrasound) and wheel encoders are placed outside this case in appropriate positions so that respective measurements can be made reliably and accurately. Schematic diagram of developed electrical systems is shown in Figure 3. The main part of the module system is a UP embedded computer [34] with Intel Atom x5 Z8350 64 bit processor and 4GB DDR3L memory and 32GB eMMC storage. It runs Ubuntu 14.04 LTS and ROS (running roscore, sensor measurement nodes as well as localization nodes). Five components are connected to the embedded computer: lidar, IMU unit, Arduino Due (for ultrasound and IR sensor data processing), motor driver (which drives the motors and communicates with wheel encoders), and joystick wireless controller. It should be noted that IMU, ultrasound, camera and IR sensors were not used as information sources in the research presented here. These sensors will be used in future development as well as in the tele-operation control scenario which is also currently under development.

Another optional part of the system is a laptop computer or tablet computer which can be used as real time visualization tools for depiction of robot's current state and planed paths. These devices can also be used to monitor battery levels, execute saved cleaning plans and/or develop new plans altogether. They are all connected to main scrubber computer via WiFi connection enabled by on board router (Asus RT-N10E) and ROS's inherently distributed design. This WiFi based configuration also allows all key components to exchange information in real time, and offers flexibility in terms of possible future upgrades. It also allowed for easier integration of UWB RTLS measurement system which ran on separate computer. However it does introduce range issues for the 


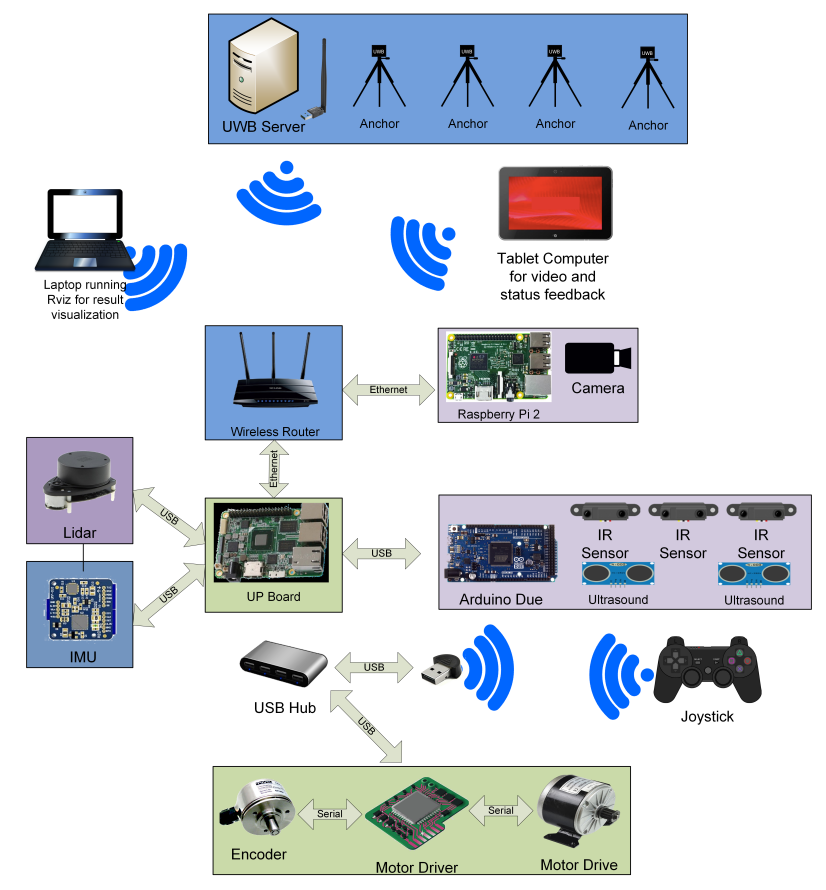

Figure 3. Schematic diagram of used electronic components and their place in the proposed prototype architecture.

scrubber (related to strength and availability of WiFi signal) when additional devices (e.g. laptop) are used.

UWB RTLS (from Sewio [35]) provides absolute 2D position of battery powered tags, and is based on DecaWave DW1000 chip. It enables indoor and outdoor positioning accuracy to about $20 \mathrm{~cm}$ in ideal (line of sight) conditions. However this system as all other UWB based systems suffers from random jumps in location estimation due to different signal paths especially in non line of sight conditions, has limited range (up to $100 \mathrm{~m}$ depending on room configuration) and has the need for synchronization which imposes certain requirements on available infrastructure and their interconnection. In order to minimize some of the issues related to pose accuracy estimation and signal jumping, three tags were placed on the scrubber (see Figure 1) and measurement correction algorithm similar to one in [36] was employed. Note that this known UWB tag configuration was also exploited in Kalman filter estimation of the pose when UWB and encoder data was fused together as will be explained latter on in subsection IId.

All electronics (except drive motor) were powered by additional battery pack $(12 \mathrm{~V}, 12 \mathrm{Ah})$ with three voltage stabilizers $(5,9$ and $12 \mathrm{~V})$. Table I contains more detailed information on used electronic components and systems. Note that all components are off-the-shelf with exception of motor driver which was built in-house and has several unique features like custom wireless communication protocol, battery charging capabilities, interrupt based communication and data pre-processing in order to reduce workload on the main computer/controller.

\section{Software and Control Algorithms}

ROS was used as a driving force behind robot's operation along with open source navigation and localization plugin
Table I

DETAILS OF ELECTRONIC COMPONENTS OF THE SYSTEMS

\begin{tabular}{l|c}
\hline Driver motor & MY1016Z2 250W \\
\hline LIDAR & RoboPeak RPLIDAR 360 \\
\hline UWB Localization subsystem & Sewio RTLS system \\
\hline Ultrasound & HS-SR04 \\
\hline IR & Sharp GP2Y0A41SK0F \\
\hline IMU & UM7-LT \\
\hline Wheel encoder & LPA3806-600BM-G5-24C \\
\hline Main controller & UP Board 4GB RAM, 32 GB eMMC \\
\hline Auxiliary controller & Arduino Due \\
\hline
\end{tabular}

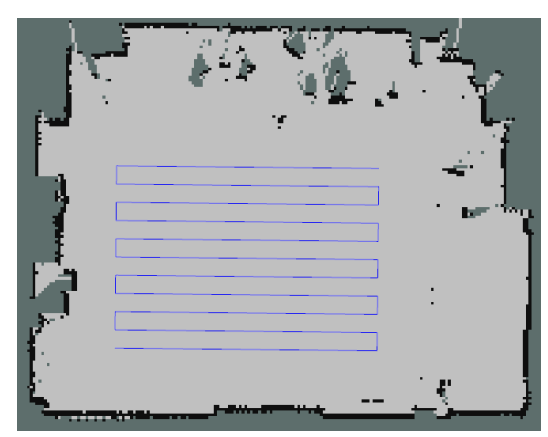

Figure 4. Example of path planning algorithm output (blue line) for cleaning a simple room.

called ROS navigation stack [37]. Navigation stack has readily available localization algorithms like Advanced Monte Carlo localization (AMCL) as well as navigation and obstacle avoidance algorithms. However, just some parts of the stack were used out of the box (like AMCL) due to specific nature of the application, while others were written from the scratch (like global and local path planning and obstacle avoidance - for simple example of global path planning algorithm see Figure 4). These algorithms and their operation are out of the scope of this article and wont be discussed further.

ROS nodes (name given to ROS based programs) for collection and processing of sensor data were written. Node associated with UWB RTLS subsystem low-pass filtered (cutoff frequency determined empirically after several test runs) UWB measurements to remove sudden jumps in robot position inherit to UWB systems (which we know is not possible due to dimensions and weight of the robot). It also removed a measurement bias observed with particular RTLS. Then it used algorithms similar to those from [36] to obtain more accurate estimation of scrubber pose. Example of results from accuracy trials after this procedure was applied are depicted in Figure 5. Gaussian like distribution can be seen in the figure with values ranging up to $15 \mathrm{~cm}$ which we take as accuracy of the system.

Additional ROS nodes were in place in order to process data from other sensors like wheel drives and output estimated position and orientation (wheel odometry) [38]. Special node based on AMCL co-variance matrix was in place for behavior-based switching i.e. determining if UWB/lidar fusion is needed, as opposed to lidar only configuration, and acting accordingly. 


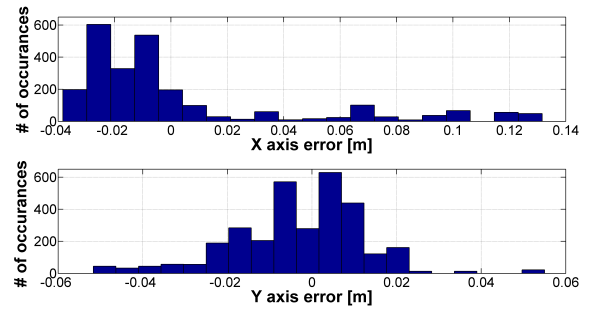

Figure 5. Example of histogram plots of UWB sensor triad accuracy after raw measurement processing and compensation.

\section{Kalman filter for UWB/wheel encoder data fusion}

Since lidar sensor still has a relatively high price and in some cases (especially in outdoor environments, and indoor environments with significant number of glass surfaces) has limited usability we wanted to explore different ways of combining UWB RTLS measurements and other sensors usually available on the robot. The decision was made to use wheel encoders since they are present on (almost) every mobile robot due to their favorable characteristics and price. For that purpose discrete Extended Kalman filter algorithm [39] was used. The algorithm used state vector defined as:

$$
\vec{x}=\left(\begin{array}{c}
x_{1} \\
y_{1} \\
x_{2} \\
y_{2} \\
x_{3} \\
y_{3} \\
v_{x_{1}} \\
v_{y_{1}} \\
v_{x_{2}} \\
v_{y_{2}} \\
v_{x_{3}} \\
v_{y_{3}}
\end{array}\right)
$$

where $x_{i}$ and $y_{i}$ are coordinates of $i-t h$ UWB tag expressed in world coordinate frame, and $v_{x_{i}}$ and $v_{y_{i}}$ are transnational speeds along $x$ and $y$ axis, respectively, for $i-t h$ UWB tag. Based on this state vector, position and speed of mobile robot's base frame could be calculated. The state vector evolved over time according to (linear) equation:

$$
\vec{x}_{k+1}=\mathbf{A} \vec{x}_{k}+\vec{w}_{k}
$$

where $\mathbf{A}$ is state transition matrix defined by system model and $\vec{w}_{k}$ is process noise (Gaussian distributed noise with mean $0)$. In particular experiment matrix $\mathbf{A}$ was defined as

$$
\mathbf{A}=\left(\begin{array}{cccccccccccc}
1 & 0 & 0 & 0 & 0 & 0 & T & 0 & 0 & 0 & 0 & 0 \\
0 & 1 & 0 & 0 & 0 & 0 & 0 & T & 0 & 0 & 0 & 0 \\
0 & 0 & 1 & 0 & 0 & 0 & 0 & 0 & T & 0 & 0 & 0 \\
0 & 0 & 0 & 1 & 0 & 0 & 0 & 0 & 0 & T & 0 & 0 \\
0 & 0 & 0 & 0 & 1 & 0 & 0 & 0 & 0 & 0 & T & 0 \\
0 & 0 & 0 & 0 & 0 & 1 & 0 & 0 & 0 & 0 & 0 & T \\
0 & 0 & 0 & 0 & 0 & 0 & 1 & 0 & 0 & 0 & 0 & 0 \\
0 & 0 & 0 & 0 & 0 & 0 & 0 & 1 & 0 & 0 & 0 & 0 \\
0 & 0 & 0 & 0 & 0 & 0 & 0 & 0 & 1 & 0 & 0 & 0 \\
0 & 0 & 0 & 0 & 0 & 0 & 0 & 0 & 0 & 1 & 0 & 0 \\
0 & 0 & 0 & 0 & 0 & 0 & 0 & 0 & 0 & 0 & 1 & 0 \\
0 & 0 & 0 & 0 & 0 & 0 & 0 & 0 & 0 & 0 & 0 & 1
\end{array}\right)
$$

where $T$ was sampling time between two state space update and in the experiment was set to wheel encoder frequency $(7.5$ $\mathrm{Hz})$. Measurement vector consisted of 15 elements and was defined as

$$
\vec{z}_{k+1}=\left(\begin{array}{c}
x_{1_{U W B}} \\
y_{1_{U W B}} \\
x_{2_{U W B}} \\
y_{2_{U W B}} \\
x_{3_{U W B}} \\
y_{3_{U W B}} \\
v_{x_{1} \text { Odom }} \\
v_{y_{1} \text { Odom }} \\
v_{x_{2} \text { Odom }} \\
v_{y_{2} \text { Odom }} \\
v_{x_{3} \text { Odom }} \\
v_{y_{3} \text { Odom }} \\
a_{\text {triangle }} \\
b_{\text {triangle }} \\
c_{\text {triangle }}
\end{array}\right)
$$

where $x_{i_{U W B}}$ and $y_{i_{U W B}}$ were coordinates of $i-t h$ UWB tag measured by UWB RTLS, $v_{x_{i} \text { Odom }}$ and $v_{y_{i} \text { Odom }}$ were transnational speeds along $x$ and $y$ axis, respectively, for $i-t h$ UWB tag as measured by wheel odometry and equations from known (and static) geometry, and $a_{\text {triangle }}, b_{\text {triangle }}$, and $c_{\text {triangle }}$ were lengths of triangle sides defined by three used UWB tags (which was known and fixed). Unfortunately, measurement update equation which relates measurement vector $\vec{z}_{k+1}$ to state space vector $\vec{x}_{k+1}$ was nonlinear (due to triangle terms which have square root for Euclidean distance), requiring linearization around last known system state. Thus EKF variant of Kalman filter was used. For detailed derivation of the EKF algorithm please refer to [39].

For the proper functioning of the whole approach equations for transforming wheel encoder readings to UWB tag speeds were of importance. In order to model the problem three coordinate frames need to be introduced as is depicted in Figure 6. Based on the figure, the following equations can be defined

$$
\alpha=\arctan \frac{Y_{\text {TAGBase }}}{X_{\text {TAGBase }}}
$$

$$
d_{T A G}=\sqrt{X_{\text {TAGBase }}^{2}+Y_{\text {TAGBase }}^{2}}
$$




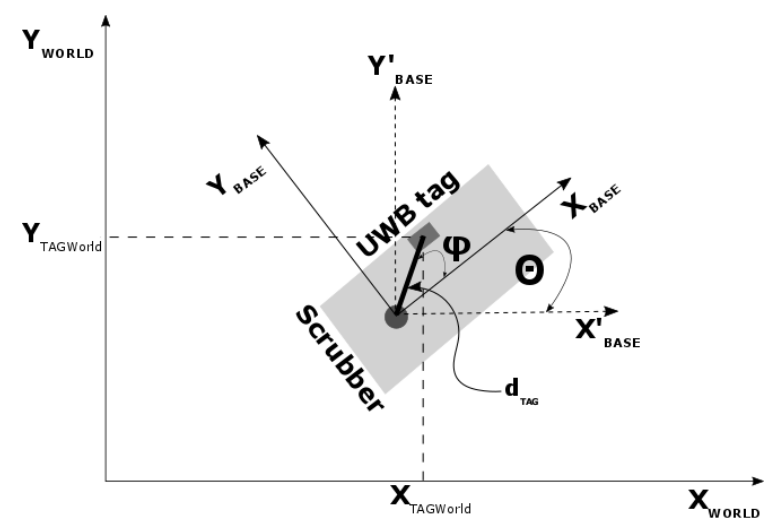

Figure 6. Coordinate frames necessary for the derivation of geometrical equations used in the Kalman filter.

$$
\begin{aligned}
& X_{\text {TAGBase }}^{\prime}=d_{T A G} \times \cos (\phi+\Theta) \\
& Y_{\text {TAGBase }}^{\prime}=d_{T A G} \times \sin (\phi+\Theta) \\
& X_{\text {TAGWorld }}=X_{0}+X \prime_{\text {TAGBase }} \\
& Y_{\text {TAGWorld }}=Y_{0}+Y_{\text {TAGBase }}
\end{aligned}
$$

In the equations $X_{T A G B a s e}^{\prime}, Y_{T A G B a s e}^{\prime}$ are coordinates of the position of UWB tag in respective coordinate frame, while $X_{0}$ and $Y_{0}$ are coordinates of scrubber's (robot's) position in world frame. From the equations, by simple time derivation respective transnational velocities can be obtained.

\section{Measurement Setup}

Accurate and reliable localization in known space is prerequisite for successful application of the module: the scrubber needs to know where it is so it knows where to go and what to clean. In order to test the performance of the proposed system, a realistic scenario was used: map of a part of fourth floor of Faculty of electrical engineering, mechanical engineering and naval architecture, Split, Croatia was made (using SLAM and mapping capabilities of ROS navigation stack) as depicted in Figure 7. Please note that in some parts of the map (e.g. long hallways) there is some distortion which is introduced during SLAM mapping process. This however does not influence correct operation of the autonomous robot under lidar localization since it can account for it. However, when working with UWB RTLS this needs to be taken into consideration. Thus special care was given to mapping spaces within UWB system's range.

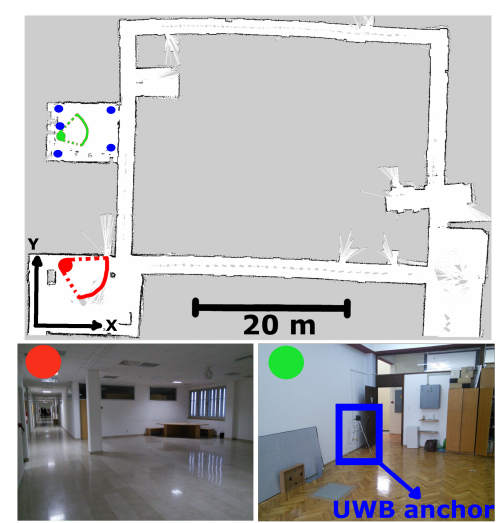

Figure 7. Part of measurement map and 3D view of selected locations. Red and green dot and lines indicate position and direction of $3 \mathrm{D}$ view in relation to the map. Blue dots represent positions of UWB anchors.

\section{A. Behavior based UWB/Lidar switching experiment}

Measurement procedure was as follows. Floor scrubber was in the known initial position and orientation. Human operator took control of the driving of the module (via wireless controller) and drove it around the map for about 15 minutes traveling in total around $200 \mathrm{~m}$ (resulting in average speed of $0.22 \frac{\mathrm{m}}{\mathrm{s}}$ ). This scenario is intended to mimic real world application where automatized floor scrubber would have to clean one floor of office type building. After completion of the lap operator parked the robot in the same position and orientation (which was previously marked on the floor). If localization measurements were ideal, robot should find itself in the same start pose on the map. Since ideal measurements are not possible, the difference between initial and final position and orientation is measured and reported (Table II). We note that knowing exact difference between robot's actual pose and pose in the map is not practical since it would require use of motion tracking hardware in all areas of measurement and it depends on SLAM performance.

For implementation of UWB and lidar behavior based switching, one room was equipped with 5 UWB anchors positioned at known locations. When scrubber was in the range (which for particular setup was about $20 \mathrm{~m}$ from master anchor) of the UWB RTLS, it's position was corrected for if predetermined conditions were met (e.g. number of lidar scan points and/or AMCL co-variance estimates). Position of UWB anchors can be seen in the upper and lower right part of Figure 7.

\section{B. UWB and wheel encoder data fusion experiment}

The same room (and UWB anchor configuration) was used for this experiment with addition of a wide angle camera (Logitech F100) which was used as a reference motion tracking system. Camera was positioned on the ceiling at approximately middle of the room (Figure 8) so that it can cover the whole space. This was in turn enabled by its wide recording angle $\left(120^{\circ}\right)$. Camera recorded with 30 frames per second with the resolution of $1920 x 1080$. Lens distortion was removed by check board calibration procedure as described in [40]. 


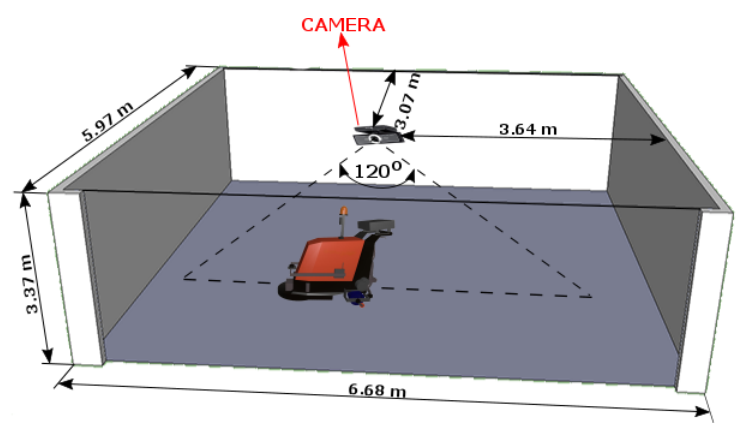

Figure 8. Sketch of measurement setup used for verification of Kalman filter performance.

The scrubber was also augmented with three color markers (of different colors - green, blue and white) so that its pose (i.e. position and orientation) can be tracked and reconstructed from video sequence. Position of the markers on the scrubber's outer shell were exactly known so that their positions can be correlated with UWB tag positions (and ultimately scrubber position i.e. base coordinate frame).

The room configuration (simple square type room) did not provide challenging environment for localization, but was the only one possible considering limitations of camera motion tracking system. In order to, at least, partially compensate for this fact complex trajectories were generated (by a human driver via wirelesses controller as in the last experiment) containing mostly eight-shape curves. Two test runs were recorded each lasting about 3 minutes and covering about 55 $\mathrm{m}$ each (resulting in the $50 \%$ increase of average speed in comparison to previous case; i.e. $0.33 \frac{\mathrm{m}}{\mathrm{s}}$ ). In these experimental runs discrepancies between actual trajectories (camera output), measured ones (UWB, odometry) and estimated ones (Extended Kalman filter) were calculated based on RMSE and not on initial/final pose difference. RMSE was defined as

$$
R M S E=\sqrt{\frac{\sum_{i=1}^{N}\left(\hat{y}_{i}-y_{i}\right)^{2}}{N}}
$$

where $N$ is total number of time instances under consideration, $\hat{y}_{i}$ is measured or estimated position along $y$ direction at $i-t h$ time instance, and $y$ is actual value at $i-t h$ time instance along the same direction. Similar equation can be derived for RMSE along $x$ direction.

\section{RESUlts AND Discussion}

\section{A. Experiment 1 - Odometry and Lidar $+A M C L$ positioning accuracy}

The odometry and lidar (via AMCL approach) measurements were taken at the same time so that their direct comparison is possible. Obtained trajectories were recorded and are visualized in Figures 9 and 10. Please note that this represents robot's belief in its current location and not actual location itself - thus through the wall trajectories are possible as in Figure 9. From the figures it is evident that considerable amount of drift is injected into odometry measurements

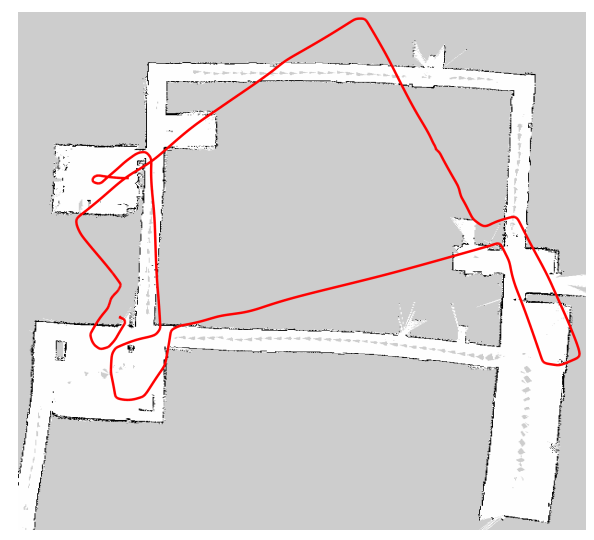

Figure 9. Robot motion trajectory as measured by wheel odometry (Odometry).

Table II

ACCURACY OF MEASURED ROBOT POSE IN UWB+LIDAR SWITCHING EXPERIMENT

\begin{tabular}{|c|c|c|c|c|}
\hline \multirow{2}{*}{ Sensor setup } & \multicolumn{3}{|c|}{ Position error [m] } & Orientation error \\
\cline { 2 - 4 } & Total & X axis & Y axis & [deg] \\
\hline Odometry & 18.96 & 8.62 & 16.89 & 38.68 \\
\hline Lidar+AMCL & 0.78 & -0.77 & -0.08 & 3.86 \\
\hline Lidar+AMCL+error & 2.22 & 0.91 & -2.03 & -15.14 \\
\hline Lidar+AMCL+UWB & 0.08 & 0.08 & 0.005 & 6.92 \\
\hline
\end{tabular}

while lidar and AMCL combination provides (more) accurate measurements.

This is confirmed by the measured error in start-end pose as presented in Table II. This in turn suggests that Lidar+AMCL is a good choice as primary localization tool for the proposed robot module. It also provides robot with good obstacle avoidance capabilities. However, we recognize that during normal operation, automatized robot scrubber might operate in open spaces (e.g. warehouses) where maximum rage of current lidar $(\leq 6 \mathrm{~m})$ will not be sufficient to maintain previously reported level of robot localization accuracy. In such situations (and space architectures) UWB based localization system might be of interest. Thus its performance needs to be tested.

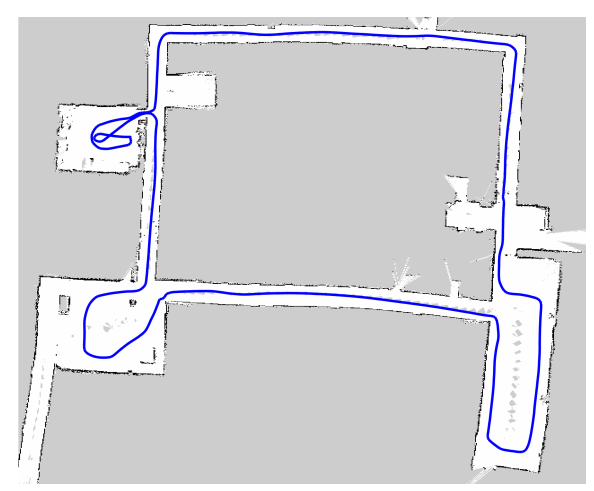

Figure 10. Robot motion trajectory as measured by lidar sensor and AMCL algorithm (Lidar + AMCL). 


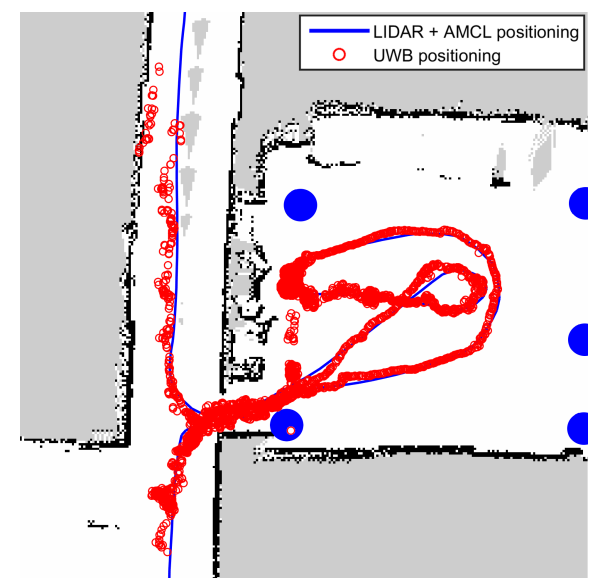

Figure 11. Comparison of robot motion trajectory as measured by lidar and AMCL approach with trajectory as measured by UWB system.

\section{B. Experiment 2 - UWB correction of Lidar and AMCL positioning}

In this experiment setup, range and accuracy of UWB RTLS was compared to lidar and AMCL combination. Obtained results are visualized in Figure 11.

From the figure it can be seen that UWB based localization matches closely that of Lidar+AMCL system. However due to significant price difference between the two systems (in favor of lidar) and infrastructure interventions required for UWB RTLS (anchor installation), lidar based system is more reasonable choice for (general) practical application. This choice is further reinforced by inspection of measurements in upper left corner of the Figure 11. Here UWB RTLS error is larger than that of lidar based system due to noideal measurement conditions (i.e. there is a wall between UWB anchors and UWB tags). Soon after, robot is out of range of UWB system and only lidar based measurements remains. However, it should be noted that such a limited range could also be in part due to WiFi signal limitations since UWB localization software was run on separate computer (due to high computational requirements) and forwarded to robot module via its WiFi router.

Nevertheless, UWB RTLS could prove to be useful in spaces with different topology (e.g. with more open spaces) where lidar and consequently AMCL performance might be sub-optimal. UWB RTLS could then offer some additional information for robot localization, despite its disadvantages. Since such spaces were not readily available at 4 th floor of the Faculty, we simulated it by putting cardboard cover over lidar module (as in Figure 12). Since lidar can not measure distances below $20 \mathrm{~cm}$ it would seem to lidar system that nothing is on that side (i.e. it is more than $6 \mathrm{~m}$ away). Robot floor scrubber was again drove on the same floor by the same operator and with same trajectory (as closely as possible). Recorded robot trajectory as estimated by Lidar+AMCL combination can be seen in Figure 12

From the figure it can be seen that in some instances (e.g. red circle) robot "goes through the wall". These instances pertain to larger open spaces to the left of the robot (since

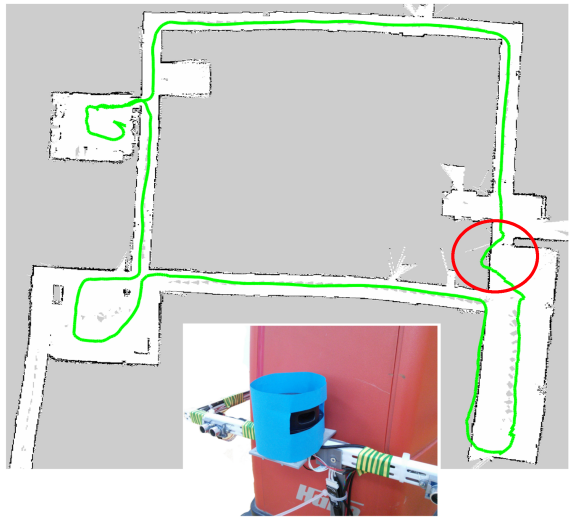

Figure 12. Lidar and AMCL trajectory estimation under simulated fault conditions (Lidar+AMCL+error). Red circle marks the area of highest AMCL algorithm's uncertainty. Smaller inner figure depicts how the error was introduced in the lidar measurements.

due to paper cover robot cannot see to its right). Trajectory was also not as smooth as before (i.e. small but frequent direction changes). Robot localization in hallways was not affected as much, although AMCL variance (and thus particle cloud) was larger than before. How this uncertainty develops (when coming from a hallway to the room with UWB anchors) can be seen in Figure 13. The further the robot goes in the open space (this was reasonably small room with approximate $7 x 7 \mathrm{~m}$ dimensions) uncertainty increases. In such situations we implemented a variant of behavior based switching algorithm. More precisely, when overall uncertainty went over predefined (experimentally determined) threshold, UWB based localization measurement (with its co-variance) is injected in the AMCL algorithm as last best estimate and the algorithm resumes from there. This is demonstrated in Figure 13: green ellipse is position estimate uncertainty after UWB based correction while red ellipse closest to it is estimate uncertainty before correction (AMCL based). Exact error in start-end pose for both cases (with and without UWB compensation) is presented in Table II (last two rows of the table).

It was also interesting to explore how AMCL uncertainty changed over time especially in larger spaces. This is depicted in Figure 14. From figure(s) it can be seen that there are several spikes in AMCL based uncertainty with the largest one both in $X$ and in $Y$ direction corresponding to highlighted red circle in Figure 12 i.e. large space. Uncertainty is smaller in hallways but is still larger than in case when no paper cover was present (i.e. without the introduced error). Figure(s) also illustrate that AMCL based uncertainty in $X$ direction (i.e. direction of robot motion) is much higher (up to 6 times) than in $Y$ direction. This might be in part to the fact that in $X$ direction there are no (or very few) distinct obstacles making accurate localization more difficult. Please note that, when talking about AMCL co-variances, $X$ and $Y$ directions are in relation to robot's coordinate frame and not the global coordinate frame as was the case before. 


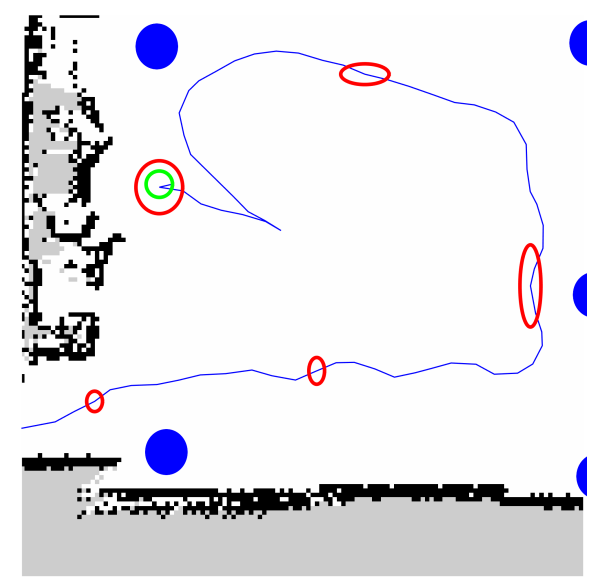

Figure 13. Lidar+AMCL based robot trajectory under simulated fault conditions and with UWB based correction (Lidar+AMCL+UWB). Red ellipses represent AMCL based uncertainty, green ellipse uncertainty after UWB based correction and blue filled circles positions of UWB anchors.
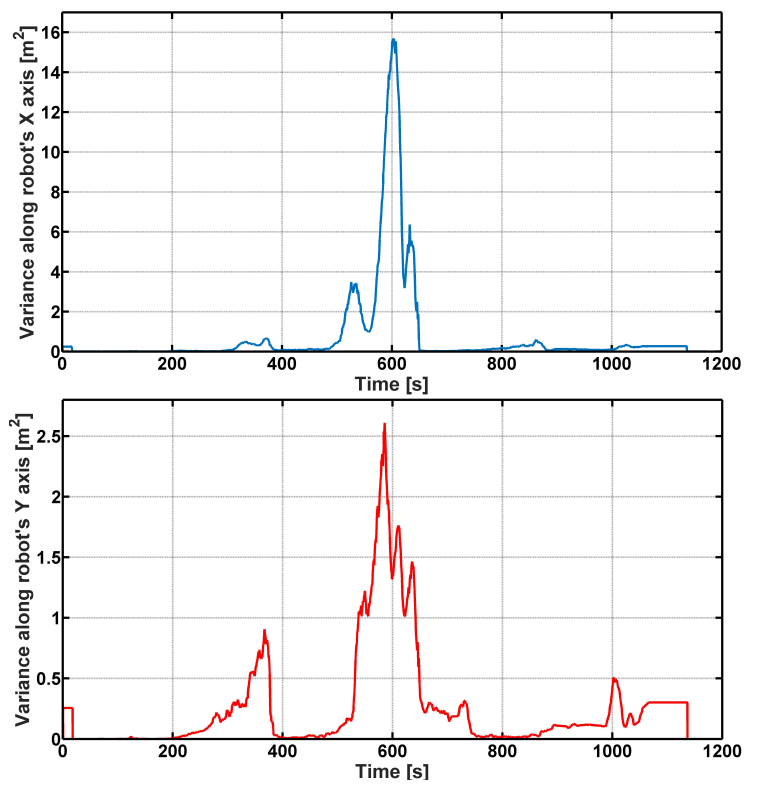

Figure 14. Variances for Lidar+AMCL combination based trajectory estimation under simulated fault conditions.

\section{Experiment 3 - UWB/Wheel encoder data fusion based on $E K F$}

In this experiment accuracy of EKF based sensor fusion of UWB RTLS and wheel encoder sensor data was examined. Summary of obtained results in terms of RMSE between trajectories for two test runs is reported in Table III.

From the table it can be concluded that wheel odometry

Table III

SUMMURIZED RMSE VALUES FOR THE EKF EXPERIMENT

\begin{tabular}{|c|c|c|c|c|c|c|}
\hline \multirow{3}{*}{ Test run } & \multicolumn{5}{|c|}{ RMSE [m] } \\
\cline { 2 - 7 } & \multicolumn{2}{|c|}{ Odometry } & \multicolumn{2}{c|}{ UWB } & \multicolumn{2}{c|}{ EKF } \\
\cline { 2 - 7 } & $\mathrm{X}[\mathrm{m}]$ & $\mathrm{Y}[\mathrm{m}]$ & $\mathrm{X}[\mathrm{m}]$ & $\mathrm{Y}[\mathrm{m}]$ & $\mathrm{X}[\mathrm{m}]$ & $\mathrm{Y}[\mathrm{m}]$ \\
\hline$\# 1$ & 0.90 & 3.16 & 0.98 & 0.83 & 0.94 & 0.78 \\
\hline$\# 2$ & 1.09 & 3.22 & 1.21 & 1.08 & 1.16 & 1.04 \\
\hline
\end{tabular}

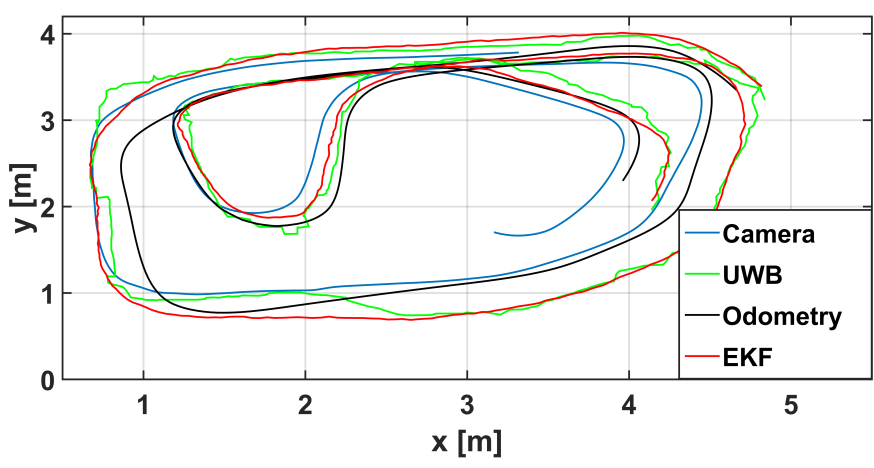

Figure 15. Example of trajectory comparisons from test run \#1 between time instances $12 s$ and $34 s$.

alone is by far the worst case scenario having error along $Y$ direction up to 3 times larger than other setups, while the error along $X$ direction is comparable or even slightly better than in other cases. Please note that $X$ and $Y$ directions here refer to global (world) coordinate frame. UWB based system improves general accuracy of robot localization compared to wheel odometry. On average it is better for $224 \mathrm{~cm}$ RMSE in $Y$ direction, but worst $10 \mathrm{~cm}$ RMSE along $X$ direction. Finally, combining wheel odometry and UWB system measurements in EKF based data fusion algorithm produces best results. The improvement over wheel odometry alone is significant, while improvement over UWB system alone is present but not as significant. To put that in actual numbers (in terms of RMSE) the average change in accuracy along $X$ directions is $-6 \mathrm{~cm}$ compared to odometry case and $+5 \mathrm{~cm}$ for UWB case, where "+" refers to increase in accuracy and "-" refers to decrease in accuracy. For $Y$ direction these changes are $+248 \mathrm{~cm}$ for odometry, and $+5 \mathrm{~cm}$ for UWB system case.

This analysis suggests that inclusion of the proposed EKF data fusion scheme does indeed increase accuracy in both directions and presents overall improvement over standalone UWB based system. For completeness, actual trajectories for all tested approaches and for one test run are presented in Figure 15. Here only about 22 seconds of experiment (out of 180) are presented since after that trajectories start to overlap and it becomes hard to distinguish different cases. Nevertheless, the figure confirms observations made from Table III. Additional fact to note when making these comparisons are different sampling frequencies: $1 \mathrm{~Hz}$ for UWB, $7.5 \mathrm{~Hz}$ for odometry and EKF based measurements, and $30 \mathrm{~Hz}$ in the case of ceiling mounted camera.

\section{CONCLUSION}

In the paper a novel add-on module which turns standard floor scrubber into a automated one (i.e. a robot) is presented. Its localization in known space is very important since the whole operation depends on it (e.g. answering the questions what and where to clean). First, three sensor configurations were tested for localization accuracy. Experimental results demonstrated that inclusion of UWB RTLS (despite its higher price and need for intervention in the navigation space through positioning of UWB anchors) is beneficial and could improve 
lidar and AMCL based localization accuracy and lower associated uncertainty in larger spaces where lidar's performance might be sub-optimal. Improvement was up to 25 times in position accuracy and 2 times in orientation accuracy. As expected odometry performance was the worst.

Afterwards it was shown that combining UWB based system with readily available sensors on mobile robots like wheel odometers can improve overall trajectory estimation accuracy in comparison to both wheel odometry and standalone UWB based system. The sensor data fusion was achieved via EKF which was computationally inexpensive and could be run in real time (although all reported results were obtained in off-line fashion). Compared to similar research ( [30], [31]) obtained results are promising and in our view demonstrate effectiveness of the proposed method despite its simplicity. When making this type of comparison it should be kept in mind that in [30] tightly coupled approach was used (as opposed to loose coupling used here) and in [31] different performance metric(s) were used. It should also be noted that proposed EKF approach yielded higher sampling frequency $(7.5 \mathrm{~Hz})$ than UWB RTLS alone $(1 \mathrm{~Hz})$.

Possible improvements of the system include upgrades in UWB based localization through coupling with Inertial Measurement Unit (IMU) via Kalman filtering approach, and UWB RTLS based SLAM mapping for more reliable maps. It should be noted that additional localization systems (like Wi-Fi based localization) can be added and easily incorporated into the proposed Extended Kalman filter structure, which should increase accuracy and reliability of the final estimate. Also other data fusion approaches like particle filtering could be tested. On the module side, inclusion of more powerful onboard computer to support navigation stack is required as well as inclusion of fully functional tele-operation subsystem which would enable off location human operator to take control in case automatic mode fails and robot does not know what to do. This is because we do recognize that human intervention could be needed in some (rare) situations when robot's navigation algorithm fails due to uncertain and dynamic nature of operational environment. Improvement of the visual design of the robot module are also planned.

\section{ACKNOWLEDGEMENT}

The work was co-funded by the "Programme of technological development, research and innovation application" (20142017) of Split-Dalmatia County, Croatia. Authors would also like to thank Odabir d.o.o. for their financial support and development of motor controller board.

\section{REFERENCES}

[1] US Department of Commerce (2017, May 10). Labor Costs [Online]. Available: http://acetool.commerce.gov/labor-costs

[2] International Organization for Standardization, ISO 8373-2012: Robots and robotic devices - Vocabulary, 2012.

[3] R. Mahoney, Service Robotics Case Studies in Silicon Valley, Silicon Valley Robotics Technical Report, 2015.

[4] Martin Hägele, Market Study on European Service Robotics, The European Robotics Coordination Action, 2013.

[5] M. Hagele, Robots Conquer the World [Turning Point], IEEE Robotics and Automation Magazine, pp.118-120, March 2016.
[6] M. Quigley and B. Gerkey and K. Conley and J. Faust and T. Foote and J. Leibs and E. Berger and R. Wheeler and A. Ng, ROS: an open-source Robot Operating System, Proceedings book of the 2009 International Conference on Robotics and Automation (ICRA) Workshop on Open Source Software, vol. 3.2, May 2013.

[7] V. Čelan and I. Stančić and J. Musić, Cleaning up Smart Cities Localization of Semi-Autonomous Floor Scrubber, Proceedings book of the 2016 International Multidisciplinary Conference on Computer and Energy Science (SpliTech), July 2016.

[8] Taski Intellibot (2017, May 10). Intellibot Products [Online]. Available: http://www.intellibotrobotics.com/products/

[9] Flobot (2017, May 10). Floor Washing Robot for Professional Users [Online]. Available: http://www.flobot.eu/

[10] B. Tribelhorn and Z. Dodds, Evaluating the Roomba: A low-cost, ubiquitous platform for robotics research and education, Proceedings book of the 2007 International Conference on Robotics and Automation (ICRA), pp. 1393-1399, April 2007.

[11] E. Ackerman and E. Guizzo, IEEE Spectrum (2017, May 10). iRobot Brings Visual Mapping and Navigation to the Roomba 980 [Online]. Available: http://spectrum.ieee.org/automaton/robotics/homerobots/irobot-brings-visual-mapping-and-navigation-to-the-roomba-980

[12] Neato Robotics, Inc., The Neato XV-11 All-Floor Vacuum System, Technical Report, 2009.

[13] E. Ackerman, IEEE Spectrum (2017, May 10). Fetch Robotics Introduces Fetch and Freight: Your Warehouse Is Now Automated [Online]. Available: http://spectrum.ieee.org/automaton/robotics/industrialrobots/fetch-robotics-introduces-fetch-and-freight-your-warehouse-isnow-automated

[14] E. Ackerman, IEEE Spectrum (2017, May 10). Clearpath's OTTO Robot Can Autonomously Haul a Ton of Stuff [Online]. Available: http://spectrum.ieee.org/automaton/robotics/industrial-robots/clearpathotto-can-autonomously-haul-a-ton-of-stuff

[15] Dispatch Robotics (2017, May 10). Dispatch robotics website [Online] Available: http://www.dispatchrobotics.com/

[16] Fellow Robotics (2017, May 10). Fellow Solutions [Online]. Available: http://fellowrobots.com/\#Solutions

[17] Omron - Adept (2017, May 10). Adept Mobile Products [Online]. Available: http://www.adept.com/products/mobile-robots

[18] Care-O-bot (2017, May 10). Care-O-bot Website [Online]. Available: http://www.care-o-bot-4.de/

[19] W. Barnett and A. Foos and T. Gruber and D. Keeling and L. Nasr, Consumer perceptions of Interactive Service Robots: A Value-Dominant Logic perspective, Proceedings book of the 23rd IEEE International Symposium on Robot and Human Interactive Communication (ROMAN), pp. 1134-1139, August 2014.

[20] A. A. Khaliq and A. Saffiotti, Stigmergy at Work: Planning and navigation for a service robot on an RFID floor, Proceedings book of the 2015 International Conference on Robotics and Automation (ICRA), pp. 1085-1092, May 2015.

[21] Robot-Era (2017, May 10). Implementation and integration of Advanced Robotic Systems and Intelligent Environments in Real Scenarios for the Aging Population [Online]. Available: http://www.robot-era.eu

[22] A. G. Ozkil, Service Robots For Hospitals: Key Technical Issues, Ph.D. Thesis, Technical University of Denmark, DTU Management Engineering, 2011.

[23] G. Baltus and D. Fox and F. Gemperle and J. Goetz and T. Hirsch and D. Margaritis and M. Montemerlo and J. Pineau and N. Roy and J. Schulte and S. Thrun, Towards Personal Service Robots for the Elderly, Proceedings book of the Workshop on Interactive Robots and Entertainment(WIRE), April/May 2000.

[24] H.T. Jung and Y.K. Choe and R. Grupen, Extended Virtual Presence of Therapists through Home Service Robots, Proceedings book of the Tenth Annual ACM/IEEE International Conference on Human-Robot Interaction (HRI) Extended Abstracts, pp. 209-210, March 2015.

[25] R. Kummerle, State Estimation and Optimization for Mobile Robot Navigation, Ph.D. Thesis, Albert-Ludwigs-Universitat Freiburg im Breisgau, Naturwissenschaften Technische Fakultat, 2013.

[26] W. Chung and G. Kim and M. Kim and C. Lee, Integrated navigation system for indoor service robots in large-scale environments, Proceedings book of the 2004 International Conference on Robotics and Automation (ICRA), pp. 5099-5104, April/May 2004.

[27] A. Linz and A. Ruckelshausen and E. Wunder and J. Hertzberg, Autonomous Service Robots for Orchards and Vineyards: $3 D$ Simulation Environment of Multi-Sensor-Based Navigation and Applications, Proceedings book of the 12th International Conference on Precision Agriculture (ICPA), pp. 2327-2334, July 2014. 
[28] Z. Sahinoglu and S. Gezici and I. Gvenc, Ultra-wideband Positioning Systems: Theoretical Limits, Ranging Algorithms, and Protocols, Cambridge University Press, New York, NY, USA, 2011

[29] S.P. Banerjee, Improving Accuracy in Ultra-Wideband Indoor Position Tracking Through Noise Modelling and Augmentation, Ph.D. Thesis, Clemson University, 2012.

[30] M. Kok and J.D. Hol and T.B. Schön, Indoor Positioning Using Ultrawideband and Inertial Measurements, IEEE Transactions on Vehicular Technology, vol. 64, no. 4, April 2015.

[31] J.A. Corrales and F.A. Candelas and F. Torres, Hybrid tracking of human operators using IMU/UWB data fusion by a Kalman filter, Proceedings book of the 3rd ACM/IEEE International Conference on Human-Robot Interaction (HRI), March 2008.

[32] J. Wang and Y. Gao and Z. Li and X. Meng and C.M. Hancock, $A$ Tightly-Coupled GPS/INS/UWB Cooperative Positioning Sensors System Supported by V2I Communication, Sensors, vol. 16, no. 944, pp. 1-16, June 2016.

[33] B. Yamauchi, Fusing ultra-wideband radar and lidar for small UGV navigation in all-weather conditions, Proceedings book of the SPIE 7692, Unmanned Systems Technology XII, 76920O, May 2010

[34] UP Board (2017, May 10). UP Board - bridge the gap [Online]. Available: http://www.up-board.org/up/

[35] Sewio (2017, May 10). Sewio website [Online]. Available: http://www.sewio.net/

[36] M. L. Ruiz de Arbulo Gubia and T. Koskinen and M. Engels an T. Lehikoinen and F. Petre, Accuracy Improving Algorithm for Wireless $3 D$ Locating Systems, Proceedings book of the 1st International Conference on Pervasive and Embedded Computing and Communication Systems (PECCS), pp. 186-190, March 2011.

[37] ROS.org (2017, May 10). ROS 2D Navigation Stack [Online]. Available: http://wiki.ros.org/navigation

[38] E. Papadopoulos and M. Misailidis, On Differential Drive Robot Odometry with Application to Path Planning, Proceedings of the European Control Conference, pp. 5492-5499, July 2007.

[39] D. Simon, Optimal State Estimation: Kalman, H Infinity, and Nonlinear Approaches, Wiley-Interscience, Hoboken, New Jersy, USA2006.

[40] R.Y. Tsai, A versatile camera calibration technique for high accuracy $3 D$ machine vision metrology using off-the-shelf TV cameras and lenses, IEEE Journal on Robotics and Automation, vol. 3, no. 4, pp. 323-344. 1987.

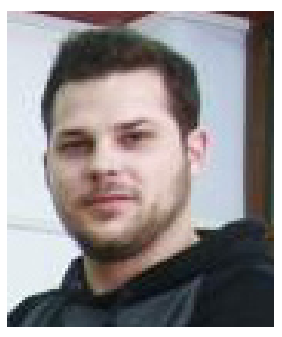

Višeslav Čelan received his Master of Engineering degree in Electronics and Computer engineering in 2011 and Master of Engineering degree in Automatic Control and Systems in 2014, both from Faculty of Electrical Engineering, Mechanical Engineering and Naval Architecture, University of Split, Croatia. He worked on project "Prototyping module for Automatic Control of Floor Scrubbers" between 2015 and 2016 within Programme of technological development, research and innovation application of Split-Dalmatia County, Croatia. From April 2016 he is employed with Statim d.o.o., Split working in their RoboLab on improving robot scrubber module. His particular research interests include localization algorithms, path planning, and obstacle avoidance algorithms as well as automatic control algorithms in mobile robotics.

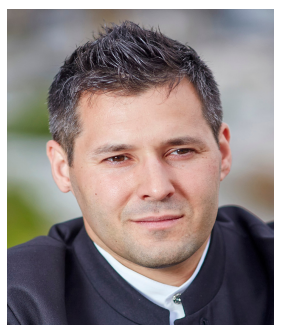

Ivo Stančić received BSc. degree in Electrical Engineering in 2006. from Faculty of Electrical Engineering, Mechanical Engineering and Naval Architecture, University of Split, Croatia. In 2012. he received $\mathrm{PhD}$ in Electrical Engineering from University of Split, Croatia. Currently, he is Assistant Professor (from 2017) in electronics at Faculty of Electrical Engineering, Mechanical Engineering and Naval Architecture, University of Split, Croatia. Prior to his current appointment, he held position of assistant/senior assistant at same Faculty since 2007. His research interests include embedded systems, 3D scanners, areal robots, and biomehcanics.

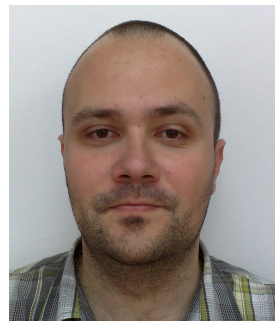

Josip Musić received BSc. degree in Electrical Engineering in 2004. from Faculty of Electrical Engineering, Mechanical Engineering and Naval Architecture, University of Split, Croatia, and MSc. degree in Electrical Engineering in 2007. from Faculty of Electrical Engineering, University of Ljubljana, Slovenia. In 2010. he received $\mathrm{PhD}$ in Electrical Engineering from University of Split, Croatia. Currently, he is Assistant Professor (from 2014) in robotics and automation at Faculty of Electrical Engineering, Mechanical Engineering and Naval Architecture, University of Split, Croatia. Prior to his current appointment, he held position of assistant/senior assistant at same Faculty since 2004. His research interests include mobile robotics, data fusion algorithms, humancomputer interaction, and rehabilitation robotics/engineering. 